\title{
Electrical carotid sinus stimulation may get lost in translation
}

\author{
Jens Jordan ${ }^{1,2} \cdot$ Jens Tank ${ }^{1,2}$ \\ Received: 26 June 2020 / Revised: 2 July 2020 / Accepted: 2 July 2020 / Published online: 22 July 2020 \\ (c) The Japanese Society of Hypertension 2020
}

There is a long rocky road from treatment targets elucidated in mechanistic studies to application in routine medical care. The translation may fail at any stage. Electrical carotid sinus stimulation showed promising efficacy in animals, including in the study by Domingos-Souza et al. published in this issue and in patients [1]. Nevertheless, the technology may be at the verge of extinction.

The arterial baroreflex is a powerful mechanism maintaining blood pressure in the face of psychological and physiological challenges. Carotid and aortic stretch receptors sense altered vascular distention as blood pressure changes. The signal is conveyed through baroreflex afferent nerves to the brainstem and integrated with input from other sources. Then, efferent cardiovascular sympathetic and parasympathetic traffic is adjusted to attenuate the blood pressure change. The baroreflex is crucial for short-term blood pressure control in humans. Damage to afferent portions of the baroreflex produces baroreflex failure with neurogenic hypertensive surges [2]. In contrast, efferent baroreflex dysfunction causes autonomic failure with disabling orthostatic hypotension [3]. The idea that baroreflex mechanisms regulate blood pressure levels in the long term is more controversial. Changes in baroreflex regulation have been observed in patients with arterial hypertension [4], which likely increases blood pressure variability, an emerging risk factor heralding excess cardiovascular risk [5]. Physiological studies suggesting that baroreflex mechanisms contribute to long-term blood pressure control [6] provided the rationale for electrical carotid sinus stimulation.

Domingos-Souza et al. [1] report findings of a study testing blood pressure responses to electrical carotid sinus

$\triangle$ Jens Jordan

jens.jordan@dlr.de

1 Institute of Aerospace Medicine, German Aerospace Center (DLR) and Chair of Aerospace Medicine, University of Cologne, Cologne, Germany

2 University Hypertension Center, University of Cologne, Cologne, Germany stimulation in rats with experimental hypertension induced through nitric oxide synthase inhibition with L-NAME. A sham group received L-NAME and underwent surgery but was not treated with electrical carotid sinus stimulation. Following one-hour baseline recordings, carotid sinus stimulation was begun using an intermittent stimulation mode. After $48 \mathrm{~h}$, the measurements were repeated for one hour. The authors also computed heart rate and blood pressure variability to assess autonomic cardiovascular control and performed ex vivo tests of explanted mesenteric resistance vessels.

L-NAME-induced substantial arterial hypertension was substantially improved by electrical carotid sinus stimulation. However, blood pressure remained within the hypertensive range. The authors observed subtle changes in heart rate and blood pressure variability, which might indicate a shift in the balance between sympathetic and parasympathetic modulation towards parasympathetic activation. Yet, the groups may have been too small to reach a more definitive conclusion, and more detailed physiological and biochemical profiling would be required to nail down the mechanism. However, the authors showed that electrical carotid sinus stimulation does not change ex vivo vascular reactivity to the alpha-adrenoreceptor agonist phenylephrine. Remarkably, carotid sinus stimulation selectively improved endothelium-dependent acetylcholine-induced vasodilation ex vivo while endothelial nitric oxide synthase protein expression or phosphorylation did not change.

The study by Domingos-Souza et al. [1] extends previous studies performed in animals [6] and in patients [7], which showed reductions in blood pressure with electrical carotid sinus stimulation. However, the study also highlights an important issue limiting the utility of the approach in routine clinical practice. Blood pressure reduction with carotid sinus simulation is at least in part mediated through sympathetic inhibition [8]. On average, sympathetic nervous system activity is increased in patients with resistant arterial hypertension [4], which is the group that is most likely to be eligible for carotid sinus stimulation. However, the sympathetic nervous system contributions to blood pressure are 
highly variable between patients [8,9]. Domingos-Souza et al. excluded animals not showing a blood pressure drop of $>20 \mathrm{mmHg}$ during stimulation from further analysis. Only one-third of patients with arterial hypertension who underwent surgical sympathectomy decades ago showed relevant chronic blood pressure improvements [10]. Thus, interventions targeting the sympathetic nervous system will not be equally effective in all patients. We have to find ways of identifying patients likely to benefit from carotid sinus stimulation before implantation.

Technological issues also limit the clinical utility of electrical carotid sinus stimulation in treating resistant arterial hypertension, namely, invasiveness, limited battery life, difficulties in optimal electrode placement for target engagement, and lack of feedback control. Baroreflex stimulators with electrodes wrapped around baroreflex afferent nerves were utilized decades ago, but their use was discontinued [11]. The more recently introduced firstgeneration electrical carotid sinus stimulator [Rheos ${ }^{\mathrm{TM}}$ ] was equipped with bilateral bipolar electrodes that were applied around the carotid sinus but is no longer available [12]. The second-generation device $\left[\mathrm{neo}^{\mathrm{TM}}\right]$ features a unilateral and unipolar disk-shaped electrode. Design evolution yielded significant reductions in invasiveness. Compared with bilateral surgery, unilateral electrode placement less commonly produces complications, such as lymph edema. Battery life has also increased but is still unsatisfactory for chronic treatment. Unfortunately, these improvements may have sacrificed efficacy [13].

Perhaps the most important reason why electrical carotid sinus stimulation may get lost in translation is the lack of data from controlled clinical trials. The first-generation device was tested in the pivotal randomized Rheos trial, which randomized 265 patients with resistant hypertension in a 2:1 fashion to device activation one month or to device activation six months following device implantation [14]. While the prospectively defined efficacy endpoint in the controlled phase of the trial, $a \geq 10 \mathrm{~mm} \mathrm{Hg}$ systolic blood pressure reduction, was not significant, blood pressure was significantly reduced. The second-generation device showed promising efficacy data in an uncontrolled trial [15]. However, a multicenter randomized controlled trial [ClinicalTrials.gov Identifier: NCT01679132] was suspended due to a lack of funding.

Despite all these challenges, we will conclude on a positive note. Research fostered by electrical carotid sinus stimulator development has had a fundamental impact on our understanding of human baroreflex regulation. In fact, the idea that baroreflex mechanisms do not fully reset and contribute to long-term blood pressure control has now been proven in animals and in human beings. Moreover, patients implanted with such devices provide insight in human physiology that could not be gained otherwise [16]. Finally, we have also learned that such treatment can effectively lower blood pressure in a subset of patients with difficult-to-control arterial hypertension. Meanwhile, novel technologies using electrical carotid sinus stimulation guided by blood pressure feedback have been shown to ameliorate hypertension and blood pressure variability in animals. Overall, there are good reasons to continue research in this area [17].

\section{Compliance with ethical standards}

Conflict of interest JJ served as an advisor for Novo-Nordisk, Eternygen, Bayer. JJ and JT received research support from BostonScientific and Boehringer Ingelheim.

Publisher's note Springer Nature remains neutral with regard to jurisdictional claims in published maps and institutional affiliations.

\section{References}

1. Domingos-Souza G, Santos-Almeida FM, Meschiari CA, Ferreira NS, Pereira CA, Martinez D, et al. Electrical stimulation of the carotid sinus lowers arterial pressure and improves heart rate variability in L-NAME hypertensive conscious rats. Hypertens Res. e-pub ahead of print 1 May 2020; https://doi.org/ 10.1038/s41440-020-0448-7.

2. Robertson D, Hollister AS, Biaggioni I, Netterville JL, MosquedaGarcia R, Robertson RM. The diagnosis and treatment of baroreflex failure. N. Engl J Med. 1993;329:1449-55.

3. Singleton CD, Robertson D, Byrne DW, Joos KM. Effect of posture on blood and intraocular pressures in multiple system atrophy, pure autonomic failure, and baroreflex failure. Circulation. 2003;108:2349-54.

4. Grassi G, Seravalle G, Brambilla G, Pini C, Alimento M, Facchetti R, et al. Marked sympathetic activation and baroreflex dysfunction in true resistant hypertension. Int J Cardiol. 2014;177:1020-5.

5. Parati G, Mancia G. Blood pressure variability as a risk factor. Blood Press Monit. 2001;6:341-7.

6. Lohmeier TE, Iliescu R. The baroreflex as a long-term controller of arterial pressure. Physiology. 2015;30:148-58.

7. Scheffers IJ, Kroon AA, Schmidli J, Jordan J, Tordoir JJ, Mohaupt MG, et al. Novel baroreflex activation therapy in resistant hypertension: results of a European multi-center feasibility study. J Am Coll Cardiol. 2010;56:1254-8.

8. Heusser K, Tank J, Engeli S, Diedrich A, Menne J, Eckert S, et al. Carotid baroreceptor stimulation, sympathetic activity, baroreflex function, and blood pressure in hypertensive patients. Hypertension. 2010;55:619-26.

9. Jordan J, Tank J, Hohenbleicher H, Toka H, Schroeder C, Sharma $\mathrm{AM}$, et al. Heterogeneity of autonomic regulation in hypertension and neurovascular contact. J Hypertens. 2002;20:701-6.

10. Grimson KS. Total thoracic and partial to-total lumbar sympathetctomy and celiac ganglionectomy in the treatment of hypertension. Ann Surg. 1941;114:753-75.

11. Rothfeld EL, Parsonnet V, Raman KV, Zucker IR, Tiu R. The effect of carotid sinus nerve stimulation on cardiovascular dynamics in man. Angiology. 1969;20:213-8.

12. Tordoir JH, Scheffers I, Schmidli J, Savolainen H, Liebeskind U, Hansky B, et al. An implantable carotid sinus baroreflex activating system: surgical technique and short-term outcome from a multicenter feasibility trial for the treatment of resistant hypertension. Eur J Vasc Endovasc Surg. 2007;33:414-21. 
13. Heusser K, Tank J, Brinkmann J, Menne J, Kaufeld J, Linnenweber-Held $\mathrm{S}$, et al. Acute response to unilateral unipolar electrical carotid sinus stimulation in patients with resistant arterial hypertension. Hypertension. 2016;67:585-91.

14. Bisognano JD, Bakris G, Nadim MK, Sanchez L, Kroon AA, Schafer J, et al. Baroreflex activation therapy lowers blood pressure in patients with resistant hypertension: results from the double-blind, randomized, placebo-controlled rheos pivotal trial. J Am Coll Cardiol. 2011;58:765-73.

15. Hoppe UC, Brandt MC, Wachter R, Beige J, Rump LC, Kroon AA, et al. Minimally invasive system for baroreflex activation therapy chronically lowers blood pressure with pacemaker-like safety profile: results from the Barostim neo trial. J Am Soc Hypertens. 2012;6:270-6.

16. Heusser K, Thöne A, Lipp A, Menne J, Beige J, Reuter H, et al. Efficacy of electrical baroreflex activation is independent of peripheral chemoreceptor modulation. Hypertension. 2020;75: 257-64.

17. Tohyama T, Hosokawa K, Saku K, Oga Y, Tsutsui H, Sunagawa K. Smart baroreceptor activation therapy strikingly attenuates blood pressure variability in hypertensive rats with impaired baroreceptor. Hypertension. 2020;75:885-92. 